\section{Oropharynx-Larynx-Tumoren: Alleinige Bestrahlung infrage gestellt}

\begin{abstract}
Bei einigen Plattenepithelkarzinomen des Kopf-Hals-Bereichs (HNSCC) ist die alleinige Bestrahlung Standard. Bislang gab es keine direkten Vergleiche zwischen Radiochemotherapie und alleiniger Strahlentherapie.
\end{abstract}

\begin{abstract}
$\mathrm{n}$ einer retrospektiven Analyse wurde die alleinige Strahlentherapie mit der Radiochemotherapie (CRT) verglichen. Basis waren Daten aus der Nationalen Krebsdatenbank der USA von 5.030 Patienten, die zwischen 2004 und 2012 die Diagnose eines invasiven Plattenepithelkarzinoms (cT1-2N1M0) von Oropharynx, Larynx oder Hypopharynx erhalten hatten und in kurativer Absicht bestrahlt worden waren. Patienten, die vor Bestrahlung operiert worden waren und für die Beobachtungsdaten fehlten, sowie Patienten, bei denen Zusammensetzung oder zeitlicher Einsatz der Chemotherapie unklar waren, wurden nicht berücksichtigt. Der Nachbeobachtungszeitraum umfasste median 56,8 Monate (95\%-Kon-
\end{abstract}

fidenzintervall [95\%-KI] 55,7-58,6 Monate). $68 \%$ der Patienten hatten eine gleichzeitige CRT (CCRT) erhalten. Der Anteil dieser Patienten war zwischen 2004 und 2012 signifikant von 53 auf $78 \%$ angestiegen $(\mathrm{p}<0,001)$.

Eine CCRT ging der multivariaten Analyse zufolge mit einem signifikant besseren Gesamtüberleben (OS) einher als die alleinige Radiatio. Die Hazard Ratio (HR) betrug 0,80 , mit einem $95 \%$-KI von $0,72-0,88(\mathrm{p}<0,001)$. In PropensityScore-adjustierten Analysen blieb die CCRT signifikant mit einem besseren OS assoziiert. Berücksichtigt wurden dabei Faktoren wie Alter, Komorbiditätsgrad, Tumorstadium, Lokalisation des Karzinoms, Diagnosejahr sowie Charakteris- tika der behandelnden Einrichtung und weitere demografische Faktoren. Die 5-Jahres-OS-Rate betrug in dieser Analyse bei CCRT 63,5\% (95\%-KI 60,7$66,2 \%$ ) und bei Radiatio alleine $55,6 \%$ (95\%-KI 52,7-58,4\%; p < 0,001). Subgruppenanalysen deuteten bei der überwiegenden Zahl von Subgruppen auf einen Vorteil der CCRT hin. Das galt auch für Patienten mit oropharyngealen Tumoren (HR 0,74; 95\%-KI 0,65-0,85; $\mathrm{p}<0,001$ ), für die das National Comprehensive Cancer Network (NCCN) in seinen Leitlinien explizit die alleinige Strahlentherapie empfiehlt.

Fazit: Die Daten weisen auf einen Überlebensvorteil bei Strahlenchemotherapie gegenüber der alleinigen Strahlentherapie hin. Deshalb regen die Forscher eine prospektive Studie zu dieser Fragestellung bei Patienten mit HNSCC (T1$2 \mathrm{~N} 1)$ an.

Friederike Klein

Zumsteg ZS et al. Impact of concomitant chemoradiation on survival for patients with T1-2N1 head and neck cancer. Cancer. 2017; 123 (9): 1555-65.

\title{
PET/CT-Scan erhöht Sicherheit der Nachsorge bei Kopf-Hals-Tumoren
}

\begin{abstract}
Bei Kopf-Hals-Plattenepithelkarzinomen (HNSCC) ist nach Abschluss der Primärtherapie die frühe Erkennung von Rezidiven für eine Salvage-Therapie und die Prognose der Patienten wichtig. In einer prospektiven Studie wurde untersucht, welche Rolle dabei die 18-Fluorodeoxyglukose-Positronenemissionstomografie (18-FDG-PET)/Computertomografie (CT) spielt.
\end{abstract}

\footnotetext{
$A^{n}$ n der Studie nahmen 278 Patienten mit HNSCC nach kurativer Operation $(\mathrm{n}=143 ; 51,4 \%)$ oder definitiver Strahlen- oder Radiochemotherapie $(\mathrm{n}=$ $135 ; 48,6 \%)$ ohne anderweitige Vorbehandlung teil. Die Nachsorge erfolgte regelmäßig mittels 18-FDG-PET/CT, CT/ Magnetresonanztomografie (MRT) von Kopf und Hals sowie Thorax-CT. Die Frequenz der Untersuchungen nahm von ein- bis dreimonatlich im ersten Jahr nach Therapieende bis auf einmal jährlich nach fünf Jahren ab. Die Befundung der Bildgebung wurde mit histologischen Ergebnissen der Rezidive verglichen. Zum Vergleich der diagnostischen Genauigkeit von 18-FDG-PET/CT und
}

einer Standardbildgebung mit CT/MRT und Thorax-CT führten die Forscher unter anderem die Grenzwertoptimierungskurven (ROC; „receiver operating characteristics curves"), den McNemarTest und eine logistische Regression durch. Für die Berechnung des prognostischen Werts von PET/CT verwendeten sie ein multivariates Cox-Risikomodell.

Über einen medianen Beobachtungszeitraum von 44 Monaten entwickelten 73 Patienten $(26,3 \%)$ ein Rezidiv oder wiesen eine persistierende Erkrankung auf. Die AUC („area under the curve“) in der ROC-Analyse betrug bei 18-FDGPET/CT 0,975 und wies dabei auf eine signifikant größere Genauigkeit hin als die AUC bei CT/MRT mit 0,789 $(\mathrm{p}<0,001)$. Der PET/CT-Scan ermöglichte es, 65 von insgesamt 66 Rezidiven $(98,5 \%)$ zu erkennen, die bei körperlicher Untersuchung und Endoskopie nicht aufgefallen waren.

Positive Befunde in einem 18-FDGPET/CT, egal zu welchem Zeitpunkt, waren unabhängige Prädiktoren eines schlechteren krebsspezifischen und Gesamtüberlebens.

Fazit: Durch die regelmäßige Kontrolluntersuchung mittels 18-FDG-PET/CT nach Abschluss der Primärtherapie eines HNSCC können Rezidive oder Residuen mit hoher Sicherheit erkannt werden. Dies hilft bei der Einschätzung der weiteren Prognose. Friederike Klein

Kim SA et al. 18F-FDG PET/CT surveillance for the detection of recurrence in patients with head and neck cancer. Eur J Cancer. 2017; 72: 62-70. 